\title{
ON PROPERTIES OF FINITE-ORDER MEROMORPHIC SOLUTIONS OF A CERTAIN DIFFERENCE PAINLEVÉ I EQUATION
}

\author{
MEI-RU CHEN ${ }^{\bowtie}$ and ZONG-XUAN CHEN
}

(Received 27 June 2011)

Abstract

In this paper, we investigate properties of finite-order transcendental meromorphic solutions, rational solutions and polynomial solutions of the difference Painlevé I equation

$$
f(z+1)+f(z)+f(z-1)=\frac{a z+b}{f(z)}+c,
$$

where $a, b$ and $c$ are constants, $|a|+|b| \neq 0$.

2010 Mathematics subject classification: primary 30D35.

Keywords and phrases: difference Painlevé equation, transcendental solution, rational solution, polynomial solution.

\section{Introduction and results}

In this paper, we assume that the reader is familiar with the basic notions of Nevanlinna's value distribution theory (see [14, 19, 24]). In addition, we use the notation $\sigma(f)$ to denote the order of growth of $f(z)$; and $\lambda(f), \lambda(1 / f)$ and $\tau(f)$ to denote, respectively, the exponents of convergence of zeros, poles and fixed points of $f(z)$. Note that the exponent of convergence of fixed points of $f$ is defined as

$$
\tau(f)=\limsup _{r \rightarrow \infty} \frac{\log N(r, 1 /(f-z))}{\log r} .
$$

Painlevé and his colleagues [21] classified all equations of Painlevé type of the form

$$
\frac{d^{2} y}{d z^{2}}=F\left(z ; y, \frac{d y}{d z}\right)
$$

This project was supported by the National Natural Science Foundation of China (Nos 10871076 and 11171119).

(c) 2011 Australian Mathematical Publishing Association Inc. 0004-9727/2011 \$16.00 
where $F$ is rational in $y$ and $d y / d z$ and (locally) analytic in $z$. The first of these is $P_{I}$ :

$$
\frac{d^{2} y}{d z^{2}}=6 y^{2}+z
$$

Differential Painlevé equations were discovered at the beginning of the last century. Although they were discovered from mathematical considerations, they occur in many physical situations-plasma physics, statistical mechanics, nonlinear waves, quantum gravity, general relativity, quantum field theory, nonlinear optics and fibre optics. Painlevé equations have attracted much interest as the reduction of soliton equations which are solvable by inverse scattering transformations (see [1, 3, 17]), and so on. In particular, Kudryashov [18] showed that the second Painlevé equation can be used as a model for describing the electric field in a semiconductor.

In the past 20 years, discrete Painlevé equations have become important research problems (see [9]). For example, the equation

$$
y_{n+1}+y_{n-1}=\frac{\alpha n+\beta}{y_{n}}+\gamma,
$$

where $\alpha, \beta$ and $\gamma$ are constants, $n \in \mathbb{N}$, is known as the special discretization of $P_{I}$.

Recently, a number of papers (including $[2,4,6,8,10-13,15,16,20]$ ) have focused on complex difference equations and difference analogues of Nevanlinna's theory. While investigating difference analogues of Nevanlinna's theory, many results on complex difference equations were rapidly obtained.

Some results on the existence of meromorphic solutions for certain difference equations were obtained by Shimomura [22] and Yanagihara [23] during the early 1980s.

Ablowitz et al. [2] looked at difference equations of the type

$$
w(z+1)+w(z-1)=R(z, w)
$$

where $R$ is rational in both of its arguments, and showed the following theorem.

THEOREM A (See [2]). If the second-order difference equation

$$
y(z+1)+y(z-1)=\frac{a_{0}(z)+a_{1}(z) y+\cdots+a_{p}(z) y^{p}}{b_{0}(z)+b_{1}(z) y+\cdots+a_{q}(z) y^{q}},
$$

where $a_{i}$ and $b_{i}$ are polynomials, admits a nonrational meromorphic solution of finite order, then $\max (p, q) \leq 2$.

Halburd and Korhonen [11-13] used value distribution theory and reasoning related to singularity confinement to single out difference Painlevé I equations from the difference equation (1.1). They obtained that if (1.1) has an admissible meromorphic solution of finite order, then either $w$ satisfies a difference Riccati equation, or (1.1) can be transformed by a linear change in $w$ to some classical difference equations, which 
include the difference Painlevé I equations

$$
\begin{gathered}
f(z+1)+f(z-1)=\frac{a z+b}{f(z)}+c, \\
f(z+1)+f(z-1)=\frac{a z+b}{f(z)}+\frac{c}{f(z)^{2}}, \\
f(z+1)+f(z)+f(z-1)=\frac{a z+b}{f(z)}+c,
\end{gathered}
$$

where $a, b$ and $c$ are constants.

From the above, we see that the difference Painlevé I equation is the development of the differential and discrete Painlevé I equation. So it is an important class of difference equation. Besides, the Painlevé I equation is important because it creates a new transcendental function. Therefore, it is important to investigate properties of finiteorder transcendental meromorphic solutions and rational solutions and polynomial solutions of this difference Painlevé I equation.

Chen and Shon [7] investigated some properties of meromorphic solutions of the difference Painlevé I equation, and proved the following theorem.

THeOREM B. Let $a, b, c$ be constants with $a \neq 0$. If $f(z)$ is a finite-order transcendental meromorphic solution of the difference Painlevé I equation (1.2), then:

(1) $f$ has at most one nonzero finite Borel exceptional value;

(2) $\lambda(1 / f)=\lambda(f)=\sigma(f)$;

(3) $\quad f(z)$ has infinitely many fixed points and satisfies $\tau(f)=\sigma(f)$.

In [7], they also consider properties of rational solutions of (1.2).

Theorem C. Let $a, b$, c be constants where $a, b$ are not both equal to zero.

(1) If $a \neq 0$, then (1.2) has no rational solution.

(2) If $a=0$, and $b \neq 0$, then (1.2) has the nonzero constant solution $f(z)=A$, where $A$ satisfies $2 A^{2}-c A-b=0$. The other rational solution $f(z)$ satisfies $f(z)=A+P(z) / Q(z)$, where $P(z)$ and $Q(z)$ are relatively prime polynomials and satisfy $\operatorname{deg} P(z)<\operatorname{deg} Q(z)$.

Chen [5] considered the other form of the difference Painlevé I equation, that is, Equation (1.3), and proved the following two theorems.

Theorem D. Let $a, b, c$ be constants with $a c \neq 0$. Suppose that $f(z)$ is a finite-order transcendental meromorphic solution of the difference Painlevé I equation (1.3). Then:

(1) $f$ has no any Borel exceptional value;

(2) if $p(z)$ is a nonconstant polynomial, then $f(z)-p(z)$ has infinitely many zeros and $\lambda(f-p)=\sigma(f)$. 
Theorem E. Let $a, b$, $c$ be constants with $c \neq 0$ and $|a|+|b| \neq 0$. Then:

(1) if $a=0$, then (1.4) has nonzero constant solution $f(z)=B$, where B satisfies

$$
2 B^{2}-b B-c=0 ;
$$

the other rational solution $f(z)$ satisfies $f(z)=B+S(z) / H(z)$, where $S(z)$ and $H(z)$ are relatively prime polynomials satisfying $\operatorname{deg} S(z)<\operatorname{deg} H(z)$;

(2) if $a \neq 0$ and $f(z)=S(z) / H(z)$ is a rational solution of (1.3), where

$$
S(z)=s z^{k}+s_{k-1} z^{k-1}+\cdots+s_{0}, \quad H(z)=h z^{t}+h_{t-1} z^{t-1}+\cdots+h_{0},
$$

where $s, s_{k-1}, \ldots, s_{0}$ and $h, h_{t-1}, \ldots, h_{0}$ are constants such that $s h \neq 0$, then

$$
t=k+1 \quad \text { and } \quad s=-\frac{c h}{a} .
$$

The main aims of this paper are to consider the properties of finite-order transcendental meromorphic solutions, rational solutions and polynomial solutions of the difference Painlevé I equation (1.4), and obtain the following results.

THEOREM 1.1. Let $a, b, c$ be constants such that $|a|+|b| \neq 0$. If $f(z)$ is a finite-order transcendental meromorphic solution of the difference Painlevé I equation (1.4), then:

(1) $\lambda(1 / f)=\lambda(f)=\sigma(f)$;

(2) if $p(z)$ is a nonconstant polynomial, then $f(z)-p(z)$ has infinitely many zeros and $\lambda(f-p)=\sigma(f)$;

(3) if $a \neq 0$, then $f(z)$ has no Borel exceptional value; if $a=0$, then the Borel exceptional value of $f(z)$ can only come from a set $E=\left\{z \mid 3 z^{2}-c z-b=0\right\}$.

Remark 1.2. Taking $P(z)=z$ in Theorem 1.1 , we immediately obtain the corresponding result that $f(z)$ has infinitely many fixed points and satisfies $\tau(f)=\sigma(f)$.

THEOREM 1.3. Let $a, b, c$ be constants such that $|a|+|b| \neq 0$.

(1) If $a \neq 0$, then (1.4) has no rational solution.

(2) If $a=0$, then (1.4) has nonzero constant solution $f(z)=B$, where $B$ satisfies $3 B^{2}-c B-b=0$. Furthermore:

(i) if $c^{2}+12 b \neq 0$, then (1.4) has no nonconstant rational solution;

(ii) if $c^{2}+12 b=0$, and $f(z)=B+S(z) / H(z)$ is a nonconstant rational solution of (1.4), where $S(z)$ and $H(z)$ are relatively prime polynomials satisfying $\operatorname{deg} S(z)<\operatorname{deg} H(z)$,

$$
S(z)=s z^{m}+s_{m-1} z^{m-1}+\cdots+s_{0}, \quad H(z)=h z^{n}+h_{n-1} z^{n-1}+\cdots+h_{0},
$$

where $s, s_{m-1}, \ldots, s_{0}$ and $h, h_{n-1}, \ldots, h_{0}$ are constants such that $s h \neq 0$, then

$$
n=m+2 \text { and } s=-\frac{c h}{3} .
$$

Remark 1.4. From Theorem 1.3, we see that if $a, b, c$ are constants and $|a|+|b| \neq 0$, then (1.4) has no nonconstant polynomial solution. 
Example 1.5. The nonzero constant solution $f(z)=B=1 / 3$ satisfies the difference Painlevé I equation (1.4)

$$
f(z+1)+f(z)+f(z-1)=\frac{1 / 6}{f(z)}+\frac{1}{2}
$$

and

$$
a=0, \quad 3 B^{2}-c B-b=0 .
$$

EXAMPLE 1.6. The nonconstant rational solution

$$
f(z)=B+\frac{S(z)}{H(z)}=1+\frac{-2}{z^{2}+3 z+2}=\frac{z^{2}+3 z}{z^{2}+3 z+2}
$$

satisfies the difference Painlevé I equation (1.4)

$$
f(z+1)+f(z)+f(z-1)=\frac{-3}{f(z)}+6
$$

and

$$
\begin{gathered}
a=0, \quad 3 B^{2}-c B-b=0, \quad c^{2}+12 b=0, \\
B=\frac{p}{q}, \quad n=m+2 \quad \text { and } \quad s=-\frac{c h}{3} .
\end{gathered}
$$

\section{Lemmas for proofs of theorems}

Lemma 2.1 (Valiron-Mohon'ko lemma (see [19])). Let $f(z)$ be a meromorphic function. Then for all irreducible rational functions in $f$,

$$
R(z, f(z))=\frac{\sum_{i=0}^{m} a_{i}(z) f(z)^{i}}{\sum_{j=0}^{n} b_{j}(z) f(z)^{j}},
$$

with meromorphic coefficients $a_{j}(z), b_{j}(z)\left(a_{m} b_{n} \not \equiv 0\right)$, the characteristic function of $R(z, f(z))$ satisfies

$$
T(r, R(z, f(z)))=d T(r, f)+o(\Psi(r)),
$$

where $d=\max \{m, n\}$, and $\Psi(r)=\max _{i, j}\left\{T\left(r, a_{i}\right), T\left(r, b_{j}\right)\right\}$. In particular, if

$$
T\left(r, a_{i}\right)=S(r, f)(i=0,1, \ldots, m), \quad T\left(r, b_{j}\right)=S(r, f)(j=0,1, \ldots, n),
$$

then we have

$$
T(r, R(z, f(z)))=d T(r, f)+S(r, f) .
$$

LEMMA 2.2 (See [20]). Let $w(z)$ be a nonconstant finite-order meromorphic solution of

$$
P(z, w)=0,
$$

where $P(z, w)$ is a difference polynomial in $w(z)$. If $P(z, a) \not \equiv 0$ for a meromorphic function a satisfying $\lim _{r \rightarrow \infty}(T(r, a) / T(r, w))=0$, then

$$
m\left(r, \frac{1}{w-a}\right)=S(r, w)
$$

outside of a possible exceptional set of finite logarithmic measure. 
LEMMA 2.3 (See [20]). Let $f$ be a transcendental meromorphic solution of finite order $\sigma$ of a difference equation of the form

$$
H(z, f) P(z, f)=Q(z, f),
$$

where $H(z, f)$ is a difference product of total degree $n$ in $f(z)$ and its shifts, and where $P(z, f), Q(z, f)$ are difference polynomials so that the total degree of $Q(z, f)$ is at most $n$. Then, for each $\varepsilon>0$,

$$
m(r, P(z, f))=O\left(r^{\sigma-1+\varepsilon}\right)+S(r, f)
$$

possibly outside of an exceptional set of finite logarithmic measure.

Lemma 2.4 (See $[12,13,20]$ ). Let $f(z)$ be a nonconstant finite-order meromorphic function. Then

$$
N(r+1, f)=N(r, f)+S(r, f)
$$

outside of a possible exceptional set of finite logarithmic measure.

\section{Proofs of theorems}

3.1. Proof of Theorem 1.1. Assume that $f(z)$ is a finite-order transcendental meromorphic solution of (1.4).

(1) First, we prove that $\lambda(f)=\sigma(f)$. By (1.4), we obtain that

$$
P(z, f):=f(z)(f(z+1)+f(z)+f(z-1))-(a z+b)-c f(z)=0 .
$$

By (3.1) and $|a|+|b| \neq 0$, we see that

$$
P(z, 0):=-(a z+b) \not \equiv 0 .
$$

Then by Lemma 2.2 and (3.2), we get that

$$
m\left(r, \frac{1}{f}\right)=S(r, f)
$$

outside of a possible exceptional set of finite logarithmic measure. Thus

$$
N\left(r, \frac{1}{f}\right)=T(r, f)+S(r, f)
$$

outside of a possible exceptional set of finite logarithmic measure. Hence, by (3.3), we have $\lambda(f)=\sigma(f)$.

Secondly, we show that $\lambda(1 / f)=\sigma(f)$. By (1.4), we obtain that

$$
f(z) G(z, f)=H(z, f)
$$

where

$$
\begin{gathered}
G(z, f)=f(z+1)+f(z)+f(z-1), \\
H(z, f)=a z+b+c f(z) .
\end{gathered}
$$


By (3.4)-(3.6) and Lemma 2.3, we see that for any given $\varepsilon>0$, there is a subset $H \subset(1, \infty)$ having finite logarithmic measure such that for $|z|=r \notin[0,1] \cup H$,

$$
m(r, G)=O\left(r^{\sigma-1+\varepsilon}\right)+S(r, f),
$$

where $\sigma=\sigma(f)$. By (1.4),

$$
G(z, f)=f(z+1)+f(z)+f(z-1)=\frac{a z+b}{f(z)}+c .
$$

By Lemma 2.1 (Valiron-Mohon'Ko lemma), and (3.8), we see that

$$
T(r, G)=T(r, f)+S(r, f) .
$$

Thus, (3.7) and (3.9) give

$$
N(r, G)=T(r, f)+O\left(r^{\sigma-1+\varepsilon}\right)+S(r, f) .
$$

On the other hand, if $f(z)$ has a pole of multiplicity $m$ at $z_{0}$ and $\left|z_{0}\right| \leq r-1$, then $f(z+1)$ and $f(z-1)$ have poles $z_{0}-1, z_{0}+1$ of multiplicity $m$, respectively. So

$$
N(r, G) \leq 3 N(r+1, f) \text {. }
$$

By Lemma 2.4,

$$
N(r+1, f)=N(r, f)+S(r, f) .
$$

Hence, by (3.10)-(3.12) we obtain that

$$
\lambda\left(\frac{1}{f}\right)=\sigma(f) .
$$

(2) Suppose that $p(z)$ is a nonconstant polynomial. We use a similar method to the above to prove that $\lambda(f-p)=\sigma(f)$. Set

$$
p(z)=d_{k} z^{k}+\cdots+d_{0},
$$

where $d_{k}, \ldots, d_{0}$ are constants, $d_{k} \neq 0$ and $k \geq 1$, and

$$
g_{1}(z)=f(z)-p(z) .
$$

Then $\lambda\left(g_{1}\right)=\lambda(f-p), \sigma\left(g_{1}\right)=\sigma(f-p)$. Substituting $f(z)=g_{1}(z)+p(z)$ into (1.4), we obtain that

$$
g_{1}(z+1)+g_{1}(z)+g_{1}(z-1)+p(z+1)+p(z)+p(z-1)=\frac{a z+b}{g_{1}(z)+p(z)}+c .
$$

By (3.14), we obtain that

$$
\begin{aligned}
P_{1}\left(z, g_{1}\right):=( & g_{1}(z+1)+g_{1}(z)+g_{1}(z-1) \\
& +p(z+1)+p(z)+p(z-1))\left(g_{1}(z)+p(z)\right) \\
& -(a z+b)-c\left(g_{1}(z)+p(z)\right)=0 .
\end{aligned}
$$


By (3.15), we see that

$$
\begin{aligned}
P_{1}(z, 0) & :=(p(z+1)+p(z)+p(z-1)) p(z)-(a z+b)-c p(z) \\
& =3 d_{k}^{2} z^{2 k}+\cdots \neq \equiv .
\end{aligned}
$$

Continuing to use the same method as above, we obtain that $\lambda(f-p)=\sigma(f)$.

(3) We prove that if $a \neq 0$, then $f(z)$ has no Borel exceptional value.

We show that for any finite value $\alpha$, we have $\lambda(f-\alpha)=\sigma(f)$. Set $g_{2}(z)=f(z)-\alpha$. Substituting $f(z)=g_{2}(z)+\alpha$ into (1.4), we obtain that

$$
g_{2}(z+1)+g_{2}(z)+g_{2}(z-1)+3 \alpha=\frac{a z+b}{g_{2}(z)+\alpha}+c .
$$

It follows from (3.16) that

$$
\begin{gathered}
P_{2}\left(z, g_{2}\right):=\left(g_{2}(z+1)+g_{2}(z)+g_{2}(z-1)+3 \alpha\right)\left(g_{2}(z)+\alpha\right) \\
-(a z+b)-c\left(g_{2}(z)+\alpha\right)=0 .
\end{gathered}
$$

By (3.16) and $a \neq 0$,

$$
P_{2}(z, 0):=3 \alpha^{2}-(a z+b)-c \alpha \not \equiv 0 .
$$

Thus by Lemma 1.3 and (3.17), we get that

$$
m\left(r, \frac{1}{g_{2}}\right)=S\left(r, g_{2}\right)
$$

outside of a possible exceptional set of finite logarithmic measure. Thus

$$
\begin{aligned}
N\left(r, \frac{1}{f-\alpha}\right) & =N\left(r, \frac{1}{g_{2}}\right) \\
& =T\left(r, g_{2}\right)+S\left(r, g_{2}\right)=T(r, f)+S(r, f),
\end{aligned}
$$

outside of a possible exceptional set of finite logarithmic measure. Hence, by (3.18), we have $\lambda(f-\alpha)=\sigma(f)$. Combining with (3.13), we see that $f(z)$ has no Borel exceptional value.

If $a=0$ and $\alpha \notin E$, then

$$
P_{2}(z, 0):=3 \alpha^{2}-c \alpha-b \not \equiv 0 .
$$

Using a similar method to the above we obtain $\lambda(f-\alpha)=\sigma(f)$. Hence, the Borel exceptional value of $f(z)$ can only come from a set $E=\left\{z \mid 3 z^{2}-c z-b=0\right\}$.

Thus Theorem 1.1 is proved.

3.2. Proof of Theorem 1.3. Assume that $f(z)$ is a nonzero rational solution of (1.4), and has poles $t_{1}, \ldots, t_{k}$. Consequently, we suppose that

$$
\frac{d_{j s_{j}}}{\left(z-t_{j}\right)^{s_{j}}}+\cdots+\frac{d_{j 1}}{z-t_{j}} \quad(j=1, \ldots, k)
$$


are the principal parts of $f(z)$ at $t_{j}$ respectively, where $d_{j s_{j}}, \ldots, d_{j 1}$ are constants, $d_{j s_{j}} \neq 0$. Thus, $f(z)$ can be represented as

$$
f(z)=\sum_{j=1}^{k}\left[\frac{d_{j s_{j}}}{\left(z-t_{j}\right)^{s_{j}}}+\cdots+\frac{d_{j 1}}{z-t_{j}}\right]+a_{0}+a_{1} z+\cdots+a_{m} z^{m},
$$

where $a_{0}, a_{1}, \ldots, a_{m}$ are constants.

We affirm that $a_{m}=\cdots=a_{1}=0$. Assume that $a_{m} \neq 0(m \geq 1)$. For sufficiently large $z$, by (3.19),

$$
\begin{gathered}
f(z)=a_{m} z^{m}(1+o(1)) ; \\
f(z \pm 1)=a_{m}(z \pm 1)^{m}(1+o(1)) .
\end{gathered}
$$

By (1.4), we obtain that

$$
(f(z+1)+f(z)+f(z-1)) f(z)=a z+b+c f(z) .
$$

Substituting (3.20), (3.21) into (3.22), we obtain that

$$
3 a_{m}^{2} z^{2 m}(1+o(1))=a z+b+c a_{m} z^{m}(1+o(1)) .
$$

Clearly, (3.23) is a contradiction since $a_{m} \neq 0$. Hence $a_{m}=0(m \geq 1)$.

(1) Suppose that $a \neq 0$. Above we have $a_{m}=0(m \geq 1)$. Now assume that $a_{0} \neq 0$. Then for sufficiently large $z$, by (3.19), we see that

$$
f(z)=a_{0}+o(1), \quad f(z \pm 1)=a_{0}+o(1) .
$$

By (1.4) and (3.24), we obtain that

$$
\left(3 a_{0}+o(1)\right)\left(a_{0}+o(1)\right)=a z+b+c\left(a_{0}+o(1)\right) .
$$

This is a contradiction since $a \neq 0$. Hence $a_{0}=0$.

Thus, $f(z)$ can be rewritten by (3.19) as

$$
f(z)=\frac{P(z)}{Q(z)},
$$

where

$$
P(z)=p z^{k}+p_{k-1} z^{k-1}+\cdots+p_{0}, \quad Q(z)=q z^{t}+q_{t-1} z^{t-1}+\cdots+q_{0},
$$

where $p, p_{k-1}, \ldots, p_{0}$ and $q, q_{t-1}, \ldots, q_{0}$ are constants such that $p q \neq 0$ and $k<t$. Then substituting (3.25) into (1.4), we obtain that

$$
\begin{gathered}
P(z+1) P(z) Q(z-1) Q(z)+P(z)^{2} Q(z+1) Q(z-1)+P(z-1) P(z) Q(z+1) Q(z) \\
=(a z+b) Q(z+1) Q(z)^{2} Q(z-1)+c P(z) Q(z+1) Q(z) Q(z-1) .
\end{gathered}
$$

Thus, since $k<t$ and $a \neq 0$, we see that the degree of the left-hand side of (3.27) does not exceed $2 k+2 t$, but the degree of the right-hand side of (3.27) is equal to $1+4 t$. This is a contradiction.

Hence, if $a \neq 0$, then (1.4) has no nonzero rational solution.

(2) Suppose that $a=0$. By observation for (1.4), we see that $f(z)=B$ is a nonzero rational solution of (1.4), where $B$ satisfies $3 B^{2}-c B-b=0$. Since $a_{m}=0(m \geq 1)$, 
we now suppose that $f(z)$ is a nonconstant rational solution of (1.4). Then $f(z)$ can be rewritten by (3.19) as the form (3.25), where $P(z)$ and $Q(z)$ satisfy (3.26) with $k \leq t$. Suppose that $k<t$. Then, substituting (3.25) into (1.4), we obtain that

$$
\begin{gathered}
P(z+1) P(z) Q(z-1) Q(z)+P(z)^{2} Q(z+1) Q(z-1)+P(z-1) P(z) Q(z+1) Q(z) \\
=b Q(z+1) Q(z)^{2} Q(z-1)+c P(z) Q(z+1) Q(z) Q(z-1) .
\end{gathered}
$$

Thus, in (3.28) there only exists one term $b Q(z+1) Q(z)^{2} Q(z-1)$ such that its degree is highest. This contradiction shows that $k=t$. Then, by (3.26) and (3.28), we obtain that

$$
\begin{aligned}
& \frac{p(z+1)^{k}+p_{k-1}(z+1)^{k-1}+\cdots+p_{0}}{q(z+1)^{t}+q_{t-1}(z+1)^{t-1}+\cdots+q_{0}}+\frac{p z^{k}+p_{k-1} z^{k-1}+\cdots+p_{0}}{q z^{t}+q_{t-1} z^{t-1}+\cdots+q_{0}} \\
& +\frac{p(z-1)^{k}+p_{k-1}(z-1)^{k-1}+\cdots+p_{0}}{q(z-1)^{t}+q_{t-1}(z-1)^{t-1}+\cdots+q_{0}} \\
& \quad=\frac{b\left(q z^{t}+q_{t-1} z^{t-1}+\cdots+q_{0}\right)}{p z^{k}+p_{k-1} z^{k-1}+\cdots+p_{0}}+c .
\end{aligned}
$$

By (3.29), we obtain that as $z \rightarrow \infty$,

$$
3 B^{2}-c B-b=0,
$$

where $B=p / q$. Hence, $f(z)$ can be rewritten as

$$
f(z)=B+\frac{S(z)}{H(z)}
$$

where $S(z)$ and $H(z)$ are relatively prime polynomials and satisfy $\operatorname{deg} S(z)<\operatorname{deg} H(z)$, $B$ is a constant satisfying $3 B^{2}-c B-b=0$,

$$
S(z)=s z^{m}+s_{m-1} z^{m-1}+\cdots+s_{0}, \quad H(z)=h z^{n}+h_{n-1} z^{n-1}+\cdots+h_{0},
$$

where $s, s_{m-1}, \ldots, s_{0}$ and $h, h_{n-1}, \ldots, h_{0}$ are constants such that $s h \neq 0$.

Furthermore, we consider the existence of nonconstant rational solutions for (1.4).

(i) We prove that if $c^{2}+12 b \neq 0$, then (1.4) has no nonconstant rational solution. Suppose that $f(z)$ is a nonconstant rational solution of (1.4). Then $f(z)$ can be rewritten by (3.30), where $S(z)$ and $H(z)$ are relatively prime polynomials and satisfy $m=\operatorname{deg} S(z)<\operatorname{deg} H(z)=n$. Substituting (3.30) into (1.4), we obtain that

$$
\begin{aligned}
&(4 B-c) S(z) H(z) H(z+1) H(z-1)+B S(z+1) H(z)^{2} H(z-1) \\
&+ B S(z-1) H(z)^{2} H(z+1) \\
&=-\left(S(z)^{2} H(z+1) H(z-1)+S(z) S(z+1) H(z) H(z-1)\right. \\
&+S(z-1) S(z) H(z) H(z+1)) .
\end{aligned}
$$

Comparing coefficients and degrees of all terms of (3.31), by $n>m$, we get that $(6 B-c) s h^{3} z^{m+3 n}$ is the highest-degree term of (3.31). 
Since $3 B^{2}-c B-b=0$ and $c^{2}+12 b \neq 0$, it follows that $B \neq c / 6$. Thus (3.31) is a contradiction for sufficiently large $z$. Therefore, if $a=0$ and $c^{2}+12 b \neq 0$, then (1.4) has no nonconstant rational solution.

(ii) If $c^{2}+12 b=0$, then by $3 B^{2}-c B-b=0$, we have $B=c / 6$. We divide this proof into three cases.

Case 1: $n=m+1$. When $n=m+1$, we have $3 n+m-1=2 m+2 n$. Comparing coefficients of the highest-degree terms of (3.31), we obtain that the highest-degree term of (3.31) is

$$
\left((18 B-3 c) s h^{2} h_{n-1}+(6 B-c) s_{m-1} h^{3}+3 s^{2} h^{2}\right) z^{m+3 n-1} .
$$

Since $B=c / 6$ and $s h \neq 0,(3.31)$ is a contradiction. Therefore, if $a=0, c^{2}+12 b=0$ and $n=m+1,(1.4)$ has no nonconstant rational solution.

Case 2: $n=m+2$. When $n=m+2$, we have $m+3 n-2=2 m+2 n$. Comparing coefficients of the highest-degree terms of (3.31), we obtain that

$$
\left(2 B s h^{3}\left(n^{2}-C_{n}^{2}+C_{m}^{2}-C_{n}^{1} C_{m}^{1}\right)+3 s^{2} h^{2}\right) z^{m+3 n-2}=\left(6 B s h^{3}+3 s^{2} h^{2}\right) z^{m+3 n-2}
$$

is the highest-degree term of (3.31). Since $c^{2}+12 b=0, a=0$ and $|a|+|b| \neq 0$, it follows that $b \neq 0$ and $c \neq 0$. Then by $B=c / 6, c \neq 0$ and $s h \neq 0$, we obtain that

$$
\frac{s}{h}=-\frac{c}{3}
$$

Therefore, if $c^{2}+12 b=0$, (1.4) has a nonconstant rational solution $f(z)$ satisfying $f(z)=B+S(z) / H(z)$. Let

$$
S(z)=s z^{m}+s_{m-1} z^{m-1}+\cdots+s_{0}, \quad H(z)=h z^{n}+h_{n-1} z^{n-1}+\cdots+h_{0},
$$

where $s, s_{m-1}, \ldots, s_{0}$ and $h, h_{n-1}, \ldots, h_{0}$ are constants such that $s h \neq 0$. Then

$$
n=m+2, \quad s=-\frac{c h}{3} .
$$

Case 3: $n>m+2$. When $n>m+2$, again comparing coefficients of the highestdegree terms of (3.31), we obtain that

$$
2 B s h^{3}\left(n^{2}-C_{n}^{2}+C_{m}^{2}-C_{n}^{1} C_{m}^{1}\right) z^{m+3 n-2}
$$

is the highest-degree term of (3.31). Since

$$
\begin{aligned}
n^{2}-C_{n}^{2}+C_{m}^{2}-C_{n}^{1} C_{m}^{1} & =\frac{1}{2}\left((n-m)^{2}+n-m\right) \\
& =\frac{1}{2}\left(\left(n-m+\frac{1}{2}\right)^{2}-\frac{1}{4}\right)>3 \neq 0
\end{aligned}
$$

and $s h \neq 0$, it follows that $B=c / 6=0$. So $c=0$. If $c=0$, by $c^{2}+12 b=0$, we have $b=0$. This contradicts the fact that $|a|+|b| \neq 0$ since $a=0$.

Therefore, if $a=0, c^{2}+12 b=0$ and $n>m+2,(1.4)$ has no nonconstant rational solution.

Thus Theorem 1.3 is proved. 


\section{References}

[1] M. J. Ablowitz and P. A. Clarkson, Solutions, Nonlinear Evolution Equations and Inverse Scattering, London Mathematical Society Lecture Note Series, 149 (Cambridge University Press, Cambridge, 1997), pp. 347-420.

[2] M. Ablowitz, R. G. Halburd and B. Herbst, 'On the extension of Painlevé property to difference equations', Nonlinearity 13 (2000), 889-905.

[3] M. J. Ablowitz and H. Segur, Solitons and the Inverse Scattering Transform (SIAM, Philadelphia, 1981), pp. 233-250.

[4] W. Bergweiler and J. K. Langley, 'Zeros of differences of meromorphic functions', Math. Proc. Cambridge Philos. Soc. 142 (2007), 133-147.

[5] Z. X. Chen, 'On properties of meromorphic solutions for some difference equations', Kodai Math. I. 34 (2011), 244-256.

[6] Z. X. Chen and K. H. Shon, 'On the zeros and fixed points of differences of meromorphic functions', J. Math. Anal. Appl. 344 (2008), 373-383.

[7] Z. X. Chen and K. H. Shon, 'Value distribution of meromorphic solutions of certain difference Painlevé equations', J. Math. Anal. Appl. 364 (2010), 556-566.

[8] Y. M. Chiang and S. J. Feng, 'On the Nevanlinna characteristic of $f(z+\eta)$ and difference equations in the complex plane', Ramanujan J. 16 (2008), 105-129.

[9] A. S. Fokas, 'From continuous to discrete Painlevé equations', J. Math. Anal. Appl. 180 (1993), 342-360.

[10] R. G. Halburd and R. Korhonen, 'Difference analogue of the lemma on the logarithmic derivative with applications to difference equations', J. Math. Anal. Appl. 314 (2006), 477-487.

[11] R. G. Halburd and R. Korhonen, 'Existence of finite-order meromorphic solutions as a detector of integrability in difference equations', Phys. D 218 (2006), 191-203.

[12] R. G. Halburd and R. Korhonen, 'Meromorphic solution of difference equation, integrability and the discrete Painlevé equations', J. Phys. A 40 (2007), 1-38.

[13] R. G. Halburd and R. Korhonen, 'Finite-order meromorphic solutions and the discrete Painlevé equations', Proc. Lond. Math. Soc. 94 (2007), 443-474.

[14] W. K. Hayman, Meromorphic Functions (Clarendon Press, Oxford, 1964).

[15] J. Heittokangas, R. Korhonen, I. Laine, J. Rieppo and K. Hohge, 'Complex difference equations of Malmquist type', Comput. Methods Funct. Theory 1 (2001), 27-39.

[16] J. Heittokangas, R. Korhonen, I. Laine, J. Rieppo and J. Zhang, 'Value sharing results for shifts of meromorphic functions, and sufficient conditions for periodicity', J. Math. Anal. Appl. 355 (2009), $352-363$.

[17] A. R. Its and Yu. V. Novokshenov, The Isomonodromic Deformation Method in the Theory of Painlevé Equations, Lecture Notes in Mathematics, 1191 (Springer, Berlin, 1986).

[18] N. A. Kudryashov, 'The second Painlevé equation as a model for the electric field in a semiconductor', Phys. Lett. A 233 (1997), 397-400.

[19] I. Laine, Nevanlinna Theory and Complex Differential Equations (Walter de Gruyter, Berlin, 1993).

[20] I. Laine and C. C. Yang, 'Clunie theorems for difference and $q$-difference polynomials', J. Lond. Math. Soc. 76(3) (2007), 556-566.

[21] P. Painlevé, 'Mémoire sur les équations differentielles dont l'intégrale générale est uniforme', Bull. Soc. Math. France 28 (1900), 201-261.

[22] S. Shimomura, 'Entire solutions of a polynomial difference equation', J. Fac. Sci. Univ. Tokyo Sect. IA Math. 28 (1981), 253-266.

[23] N. Yanagihara, 'Meromorphic solutions of some difference equations', Funkcial. Ekvac. 23 (1980), 309-326.

[24] L. Yang, Value Distribution and New Research (Science Press, Beijing, 1982) (in Chinese). 
MEI-RU CHEN, School of Mathematical Sciences,

South China Normal University, Guangzhou, 510631, PR China e-mail: chenmeiru2005@163.com

ZONG-XUAN CHEN, School of Mathematical Sciences, South China Normal University, Guangzhou, 510631, PR China e-mail: chzx@vip.sina.com 